\title{
Insulator-to-metal crossover induced by local spin fluctuations in strongly correlated systems
}

\author{
Luis Craco \\ Instituto de Física "Gleb Wataghin" - UNICAMP, C.P. 6165, 13083-970 Campinas - SP, Brazil
}

(October 24, 2018)

\begin{abstract}
We study the simplified Hubbard $(\mathrm{SH})$ model in the presence of a transverse field in the infinite dimension limit. The relevant one-particle Green's functions of the model are obtained by means a perturbative treatment of the hopping and of the transverse field around the atomic limit. We consider an analytical solution for the impurity problem. It is shown that this solution is very accurate in describing the spectral properties of the heavy-particles of the SH for intermediate and strong values of the on-site Coulomb interaction $U$. We find that for large values of $U$ an insulatormetal transition takes place as a function of the transverse field. We analyze the metallic phase through the behavior of the density of states and of the optical conductivity and static resistivity. Our results for the latter quantity agree with what is observed in experiments on $\mathrm{Bi}_{2} \mathrm{Sr}_{2} \mathrm{CuO}_{y}$.
\end{abstract}

\section{INTRODUCTION}

In the last few years, several experimental works have considered the problem of the magnetic response in strongly correlated electronic systems. For example, the spin blockade problem in quantum dots in magnetic fields has been considered by different groupst 2. The farinfrared transmission in thin films of $\mathrm{YBaCu}_{4} \mathrm{O}_{7}$ has been measured by Drew et a 3 and Lihn et al. $\mathbf{A}$, and a magneto-optical study of the magnetization for this com-

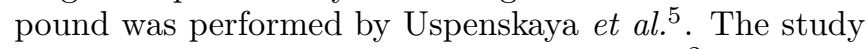
of the magnetic properties of quantum dots as well as high- $T_{c}$ thin films fields has been also of great interest.

An important problem related with high- $T_{c}$ compounds is to understand the normal state properties of the in-plane $\rho_{a b}$ and and out-of-plane $\rho_{c}$ dc-resistivity for different values of the doping concentrationl. It is well known that the easiest way to suppress the superconductivity at low temperatures without deliberate chemical substitution is to apply a high (pulsed) magnetic field. In such experiments the field is usually applied parallel to the $c$-axis to most effectively suppress the superconducting state, so that the magnetic field is normally applied perpendicular to the $\mathrm{CuO}_{2}$ bagal plane where the ordered spins are primarily aligned 10.11 . This alignment is usually originated by spin-orbitinteraction and it has been observed on different alloy 12 .

On the other hand, extensive experimental investigations show that the normal state properties of high- $T_{c}$ superconductors arenpt explained in terms of the Fermiliquid (FL) theorys $\mathrm{B}$. Deviations from the normal FL behavior were observed also in the normal state of different heavy-fermion compounds 14 as well as in quasione-dimensional materials 15 . This unexpected behavior increased the interest in studying physical models that clearly present non-Fermi liquid (NFL) properties. For example, Si et al. 16 introduced a spinless two-band model to describe the effect of interactions in a band insulating system. By solving the model numerically in the limit of infinite spatial dimensions $(d \rightarrow \infty)$ they showed that if exhibits NFL properties. In addition, Consiglio at al. 17 have shown that the main features of the optical conductivity of the Kondo alloy $Y_{1-x} U_{x} P d_{3}$ are well taken into account by the simplified Periodic Anderson model. In the last years, attention has also been given to the simplified Hubbard (SH) 18 . This is a modification of the Hubbard model, where electrons with one particular spin orientation do not hop in the lattice. This is one of the few models which possess exact solution in the limit of high dimensions 11 , and it shows a metal-insulator transition both as a function of the on-site Coulomb interaction and of the doping.

Of particular importance here is the work of Brandt and Urbanek 23 , where the spectral properties of the heavy-particles, the electrons that do not hop, are discussed in details. In addition, some years ago the $\mathrm{SH}$ model in a magnetic field has been studied by van Dongen and Leinung24. They consider the problem of the metal-insulator transition as a function of the Zeeman (z-direction) field. As expected, at large fields the system is a fully polarized ferromagnet, however in the nonsaturated phase a metal-insulator (MI) transition takes place as a function of the field and the local one-site Coulomb interaction $(U)$. They show that magnetic field slightly reduces the critical value of the Coulomb interaction, so that the MI transition for non-zero field occurs at a critical $U$ smaller then the one at zero field.

It is worth noticing that, despite of the great interest in strongly correlated electron systems, to the best of our knowledge there is no investigation of the effects of transverse fields in models which explicitly shows both broken spin symmetry and non-Fermi liquid properties. In this paper, we address precisely to this issue: the study of the $\mathrm{SH}$ model in the presence of a transverse field in the the high dimension (D) limit. This limit, introduced originally by Metzner and Vollhardt25, has been shown to be a very good_sfarting point in the study of several physical systems 26. We study the SH model because it has 
by construction broken spin symmetry, which is closely connected to the different dynamics of the two types of electrons. Here, we discuss the formal solution of this model in a transverse field, where the so called static approximation 27 is employed to solve the related singlesite problem. Comparing with the results of Ref. [23], we show that the static approximation gives a very accurate description of the $T=0$ physical properties of the heavy-particles for on-site Coulomb interactions above the metal-insulator transition. Next, we study the effect of the transverse field on the spectral and the optical properties of the SH model.

\section{MODEL AND PERTURBATION METHOD}

In the usual notation, the $\mathrm{SH}$ model in a transverse field is described by the Hamiltonian

$$
\begin{aligned}
H & =\sum_{\mathbf{k}} \epsilon_{\mathbf{k}} c_{\mathbf{k} \uparrow}^{\dagger} c_{\mathbf{k} \uparrow}+U \sum_{i} n_{i \uparrow} n_{i \downarrow}+(E-\mu) \sum_{i \sigma} n_{i \sigma} \\
& +\sum_{i} t\left(c_{i \uparrow}^{\dagger} c_{i \downarrow}+\text { h.c. }\right)
\end{aligned}
$$

where $\epsilon_{\mathbf{k}}$ is the dispersion relation of the conduction (个-electrons), and the $\downarrow$-electrons do not hop in this model. $E$ is the energy level of the two particles, which are coupled through the correlation $U$ and a transverse field $t$. This field is usually a magnetic field along the $x$ direction, but similar type of effect could have a different origin. One example would be a correlated hopping with spin flip originated in the spin orbit interaction, and this type of term has been already employed 28 in the study of other strongly correlated electron systems.

In this work we are interested in the two relevant oneparticle Green's functions of Eq. (1). The temperaturedependent one-particle Green's function for both up and down electrons are obtained, following the approach recently introduced to study the formal solution of a spinless two-band mode2 29 , by means a perturbative treatment around the atomic limit of the hopping and the transverse field terms. To apply this method, we first consider the exact solution of the unperturbed Hamiltonian, given by the last two terms of the first line of Eq. (1). Next we solve the $t=0$ limit of Eq. (11) by means of a tight-binding treatment around the atomic limit of the conduction electrons 30 . The formal solution of the complete Hamiltonian is then obtained by performing a perturbative treatment on the hybridization term²9. Following this procedure and considering the high dimension limit, it is straightforward to show that

$$
G_{i i \uparrow}\left(i \omega_{n}\right)=\frac{1}{N} \sum_{\mathbf{k}} \frac{1}{\left[\mathcal{G}_{\uparrow}\left(i \omega_{n}\right)\right]^{-1}-\epsilon_{\mathbf{k}}-t^{2} \mathcal{G}_{\downarrow}\left(i \omega_{n}\right)}
$$

and

$$
G_{i i \downarrow}\left(i \omega_{n}\right)=\mathcal{G}_{\downarrow}\left(i \omega_{n}\right)\left[1+\mathcal{G}_{\downarrow}\left(i \omega_{n}\right) t^{2} G_{i i \uparrow}\left(i \omega_{n}\right)\right],
$$

where $\mathcal{G}_{\uparrow}\left(i \omega_{n}\right)$ and $\mathcal{G}_{\downarrow}\left(i \omega_{n}\right)$ are the irreducible oneparticle Green's functions for the conduction electrons and for the heavy-particles, respectively. The former is irreducible in the sense that the contributing diagrams can not be divided in two pieces by cutting a single hopping line, while the second is irreducible with respect to the cutting a $t^{2} \bar{g}_{\mathbf{k} \uparrow}\left(i \omega_{n}\right)$ line 17 , 29 , where $\bar{g}_{\mathbf{k} \uparrow}\left(i \omega_{n}\right)$ is the solution of Eq. (11) in the limit of $t=0$. From Eq. (2) it becomes clear that the transverse field $t$ acts as an hybridization term, mixing the the singe-site one-particle excitation of the $\uparrow$-electrons with the $\downarrow$-ones. One should notice that in the case of complete Hubbard model a similar equation for the $\downarrow$-electrons is obtained by exchanging the spin directions. The main difference between Eqs. (2) 3) and those of Ref. [29] is that here they describe the formal solution of one band model with hybridization in the spin sector, while there we considered a two band model with interaction and hybridization in the charge degrees of freedom of fully polarized orbitals 31 .

The irreducible propagators in Eqs. (243) can be written in terms of the single-site one-particle Green's function and the dynamical mean-field $\mathcal{A}_{\sigma}\left(i \omega_{n}\right)$, which connects a single-site with the electron bath, through the relation 27

$$
\frac{1}{\mathcal{G}_{\sigma}\left(i \omega_{n}\right)}=\frac{1}{G_{i i \sigma}\left(i \omega_{n}\right)}+\mathcal{A}_{\sigma}\left(i \omega_{n}\right) .
$$

It is important to notice that once we turn on the transverse field, the solution of the conduction electron local problem is not trivial any longer. This is because the transverse field term hybridizes locally the up and down electrons, so that the spin-down electrons are not any longer frozen. Once we provide dynamics to the heavyelectrons, the local spin fluctuation problem also holds for the conduction electrons.

Now we proceed to analyze the solution of the singlesite problem of both electrons from the point of view of the perturbation around the atomic limit27.29. This method provides a direct way of solving the local problem by means of a perturbative expansion in the local mean-field. This approach has been used in Ref. [27] to obtain the exact solution for the conduction electrons of the SH model as well as to study the problem of the spin fluctuation in the Hubbard model. For the latter case the static approximation has been introduced. Here, we will employ this approximation to account for the local spin fluctuations induced by the transverse field.

In our perturbation approach to the single-site problem we start with the unperturbed local Green's function $-\left\langle\hat{T} c_{\sigma}(\tau) c_{\sigma}^{\dagger}(0)\right\rangle_{0}$, each order in perturbation theory introduces a product of the type $\mathcal{A}_{\sigma_{1}}\left(\tau_{1}-\tau_{1}^{\prime}\right) c_{\sigma}\left(\tau_{1}\right) c_{\sigma}^{\dagger}\left(\tau_{1}^{\prime}\right)$ so that in general one has to calculate averages of the form $\left\langle c_{\sigma_{1}}\left(\tau_{1}\right) c_{\sigma_{1}}^{\dagger}\left(\tau_{1}^{\prime}\right) c_{\sigma_{2}}\left(\tau_{2}\right) c_{\sigma_{2}}^{\dagger}\left(\tau_{2}^{\prime}\right)\right\rangle_{0}$. To calculate this averages we rewrite the fermions operators in terms of the Hubbard operators 30 , and utilize the standard algebra for the latter. This allows us to perform all possible direct contractions, in the sense of Wick's theorem. We have applied this procedure in Refs. $[27,30]$ to evaluate explicitly 
a four-operator average that appears in the one-loop approximation. In addition, in Ref. [27] the static approximation for the single-site one-particle Green's function has been obtained by neglecting all terms that involve non-zero bosonic frequencies originated by local contractions between boson like Hubbard operators. Since the averages we need to consider here are exactly the same as in the case of the Hubbard model we refer the reader to Refs. $[27,30]$ for their calculation.

In terms of the static approximation the single-site oneparticle Green's function is given by

$$
G_{i i \sigma}\left(i \omega_{n}\right)=\bar{G}_{i i \sigma}\left(i \omega_{n}\right)+\Delta_{\sigma}\left(i \omega_{n}\right),
$$

where

$$
\bar{G}_{i i \sigma}\left(i \omega_{n}\right)=\left(1-\left\langle n_{\bar{\sigma}}\right\rangle\right) \bar{g}_{0 \sigma}\left(i \omega_{n}\right)+\left\langle n_{\bar{\sigma}}\right\rangle \bar{g}_{\bar{\sigma} 2}\left(i \omega_{n}\right)
$$

is the well known exact solution for the conduction electrons of the SH model (the $t=0$ limit of Eq. (11)) and

$$
\begin{aligned}
\Delta_{\sigma}\left(i \omega_{n}\right) & =\left(g_{0 \sigma}-g_{\bar{\sigma} 2}\right) \mathcal{A}_{\bar{\sigma}}\left[\frac{\left(1-\left\langle n_{\bar{\sigma}}\right\rangle\right) \bar{g}_{0 \sigma}}{1-\mathcal{A}_{\sigma} g_{0 \sigma}-\mathcal{A}_{\bar{\sigma}}\left(g_{0 \sigma}+g_{\bar{\sigma} 2}\right)}\right. \\
& \left.-\frac{\left\langle n_{\bar{\sigma}}\right\rangle \bar{g}_{\bar{\sigma} 2}}{1-\mathcal{A}_{\sigma} g_{\bar{\sigma} 2}-\mathcal{A}_{\bar{\sigma}}\left(g_{0 \sigma}+g_{\bar{\sigma} 2}\right)}\right] .
\end{aligned}
$$

$g_{0 \sigma}\left(i \omega_{n}\right)$ and $g_{\bar{\sigma} 2}\left(i \omega_{n}\right)$ in Eqs. (6 6 团) are the fermionic Green's functions of Hubbard operators $\beta$, while $\bar{g}_{0 \sigma}\left(i \omega_{n}\right)$ and $\bar{g}_{\bar{\sigma} 2}\left(i \omega_{n}\right)$ describe the single-site one-particle excitations renormalized by the dynamical mean-field 33 . Note that even for the lowest order in the dynamical meanfield, $\Delta_{\sigma}\left(i \omega_{n}\right)$ is obtained from local contractions between one-band operators with different spin orientations. This means that $\Delta_{\sigma}\left(i \omega_{n}\right) \overline{0} 0$ for systems where local spin fluctuations are absent 31 .

\section{RESULTS}

Let us consider first the $t=0$ limit of Eq. (11). $\mathcal{A}_{\downarrow}\left(i \omega_{n}\right)=0$ in this limit, because $\mathcal{A}_{\downarrow}$ is proportional to the hybridization term. This can be easily seen from Eqs. (3) and (何. Therefore, in this limit the singlesite one-particle Green's function of the heavy-electrons $G_{i i \downarrow}\left(i \omega_{n}\right)$ is only a function of the dynamical mean-field of the conduction electrons, see Eqs. (5F). In Fig. 1 the density of states (DOS) of the heavy-electrons is shown for $t=0$ and different values of $U$ B . For $U=0.9$ we obtain a sharp peak around the Fermi level $(\omega=0)$. Increasing $U$, the height of the central peak is reduced, and a small tendency to form a gap is observed for $U=1$. From the comparison between our results with those of Ref. [23] it becomes clear that the static approximation is able to recover the main features of the spectral properties of the heavy-particles in the metallic phase of the $\mathrm{SH}$ model. Indeed, in the regime of strong on-site interaction $U$ we observe a very good agreement between our results and those obtained by Brandt et al 23 . Hence, one can conclude that the static approximation is very accurate in describing the $\downarrow$-electrons properties of the SH model in the strong coupling limit.

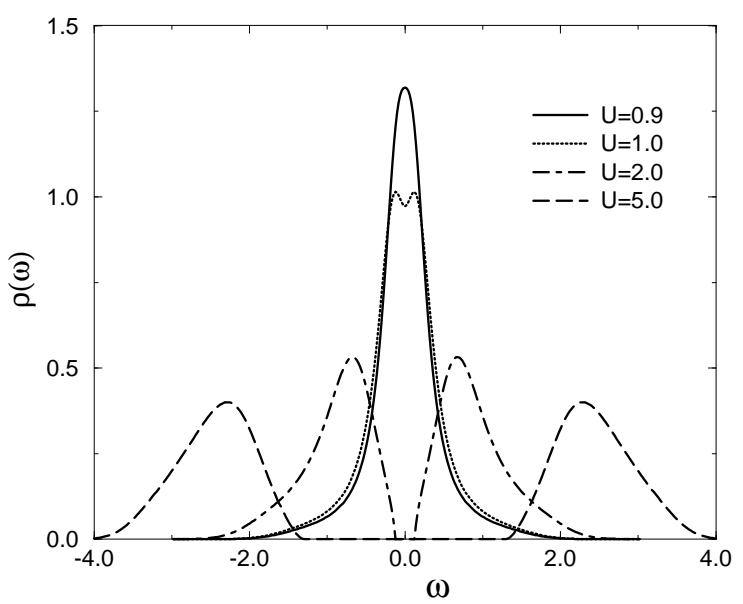

FIG. 1. Zero temperature densities of states for the heavy-electrons of the simplified Hubbard model at $t=0$ for different values of $U$.

From this result it is possible to conclude that for the half-filled and symmetric SH model at $T=0$ all the terms that involve non-zero bosonic frequencies 232730 in the local Green's functions do not play an important role in the limit of large values of the Coulomb on-site interaction. Indeed, Fig. I shows that the perturbation treatment around the atomic limit is very accurate in the large $U$ limit. Note that, in our treatment for the corrections due to spin fluctuations (Eq. (7)) an infinite series of diagrams for the site-diagonal Green's function as well as for the irreducible propagators are considered. Furthermore, based on the results of Fig. 1 it becomes clear that our solution for the single site problem is a good approximation, and we do not need to consider any small parameter to justify Eq. (7).

As our method describes correctly the spectral properties of the SH model in the large $U$ limit, we shall employ this limit to study the spectral properties of the $\mathrm{SH}$ model in the presence of the transverse magnetic field, expecting to obtain realistic results. In Fig. 2 the DOS of both types of electrons is shown for $U=2$ and different values of the transverse field $t$. Let us consider once more the $t=0$ limit. In the dashed line of Fig. 2 one can clearly see that the gap size for both particles coincide. Hence, it is clear from this behavior that the DOS at the Fermi level vanishes at the same value of $U$ for both spin directions. Furthermore, the DOS of both particles shows very strong similarities. One can expect that these similarities will increase with $U$, and that both particles would behave almost in the same way at the half-filled case for $U \rightarrow \infty$.

According to our results in Fig. 2, the charge Mott gap is strongly affected by the transverse field. In the 
case of $U=2$, this gap persists up to $t=0.3$, where the insulator-metal transition take place. It is instructive to comment that for both spin directions we observe spectral transfer from the high to low energies. The most interesting behavior of the metallic phase occurs for $t=0.5$, where a sharp peak around the Fermi level is observed in the DOS of the heavy particles. This peak is related with a self-consistent modification of the electron bath, where a partial decoupling of the local degrees of freedom take place 16 . Note that, for both nonzero values of the transverse field in Fig. 2 the two atomic-like poles of the $G_{i i \uparrow}$ remain almost in the same position, while the internal pole of the $G_{i \downarrow \downarrow}$ is shifted to zero-energy for $t=0.5$. This is because the transverse field strongly mixes the low energy excitations of the two types of electrons by spin flip process.

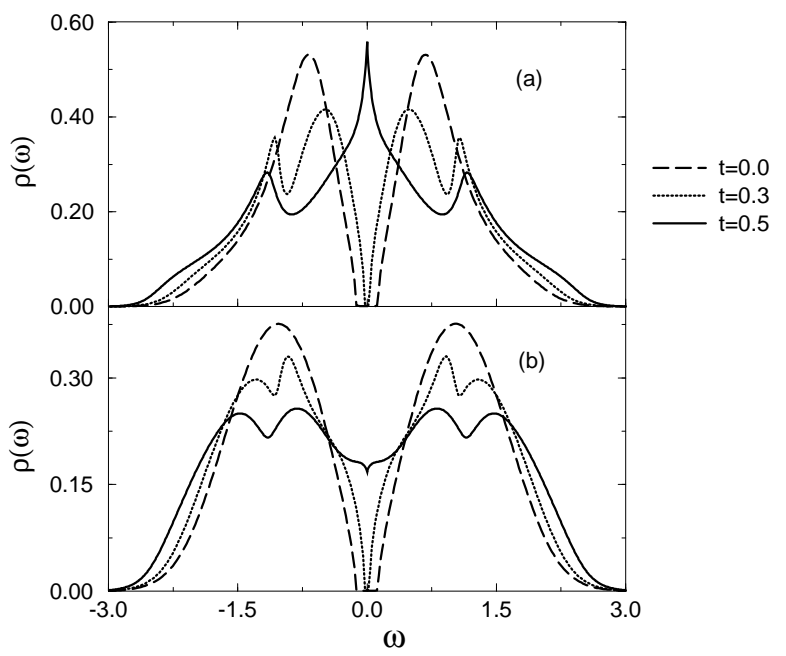

FIG. 2. Zero temperature densities of states for the SH model for $U=2$ and different values of $t$. (a) and (b) are the DOS of $\downarrow$ and $\uparrow$-electrons, respectively.

To provide a complete description of the insulatormetal transition of the $\mathrm{SH}$ model in a transverse field we study in Fig. 3 the single-particle density of states away from half-filling, at a fixed $n=0.8$. For $t=0$ one can see that our results for the DOS of the conduction electrons agrees with those obtained by Möller et al. 35 . As in Fig. 2 the gap in the DOS of both particles coincide in this limit. However, the upper Hubbard band of the heavy-electrons is higher than the one for the conduction electrons, and this behavior is related with the atomiclike character of the heavy-particles at large $U$. Another interesting feature to be seen in Fig. 3 is the presence of a second sharp peak at high energies in the DOS of the $\downarrow$ electrons for non-zero $t$. This peak reduces by increasing the transverse field. Finally, the DOS of the conduction electrons also shows interesting new structures, in particular for $t=0.5$. We believe that these structures are related with the asymmetric behavior in the DOS of the heavy-electrons together with the large hybridization between the two types of electrons.

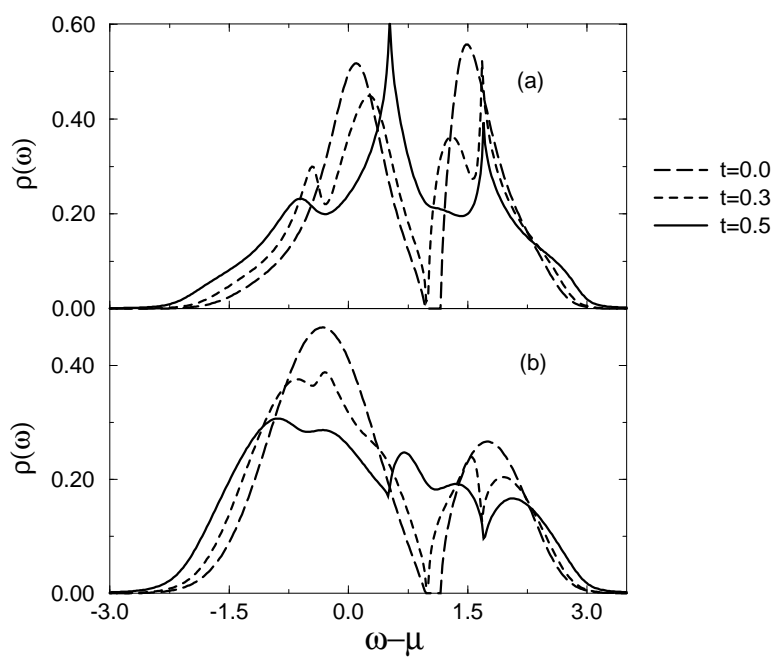

FIG. 3. Zero temperature densities of states for the $\mathrm{SH}$ model for $U=2, n=0.8$ and different values of $t$. (a) and (b) are the DOS of $\downarrow$ and $\uparrow$-electrons, respectively.

To further confirm the insulator-metal transition we calculate the optical conductivity for the half-filled case with the same parameters employed in Fig. 2. It is known that in the infinite dimension limit the vertex corrections drop out in the two particle equation, and the optical conductivity assumes the simple form 26

$$
\begin{aligned}
\sigma(\omega)= & \pi \sum_{\sigma} \int d \epsilon \rho_{0}(\epsilon) \int d \omega^{\prime} A_{\sigma}\left(\epsilon, \omega^{\prime}\right) A_{\sigma}\left(\epsilon, \omega^{\prime}+\omega\right) \\
& \times \frac{\left[f\left(\omega^{\prime}\right)-f\left(\omega^{\prime}+\omega\right)\right.}{\omega}
\end{aligned}
$$

where $\rho_{0}(\epsilon)$ is the uncorrelated density of states of the conduction band, $f(\epsilon)$ is the Fermi function and $A_{\sigma}\left(\epsilon, \omega^{\prime}\right)$ is the one-particle spectral density of the total conduction-electrons Green's function.

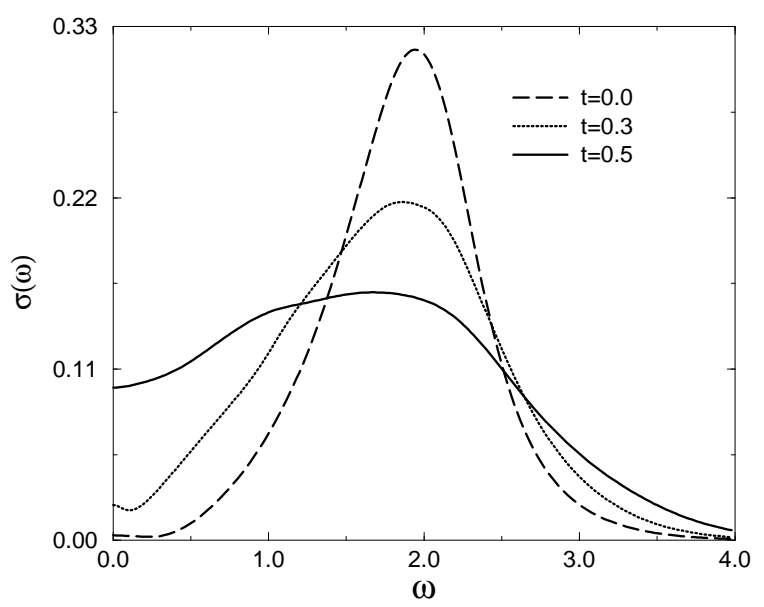

FIG. 4. Zero temperature optical conductivity for the SH model in the half-filled case for $U=2$, and three different values of the applied transverse magnetic field. 
Our results for the optical conductivity are shown in Fig. 4. For $t=0$ we have a very good agreement with those of Ref. [35]. As one can see, the system is insulator with a large gap in the optical conductivity and the maximum value of $\sigma(\omega)$ coincides with the value of the Hubbard interaction. Once we turn on the transverse field, we observe a spectral transfer from high to low energies, as in the DOS. The spectral transfer closes the gap and increases the conductivity at low frequencies. As we expect the system is a incoherent metal (no Drude peak feature is found) mainly because the transverse field is not able to completely restore the dynamics of the heavy-particles.

Now we turn our attention to Fig. 5 where we show our results for the dc-resistivity at half-filling as a function of the temperature. In $d=\infty$ this quantity is given by the inverse $(1 / \sigma(0))$ of the static limit $(\omega=0)$ of Eq. (8)

$$
\sigma(0)=\frac{\pi}{T} \int d \epsilon \rho_{0}(\epsilon) \int d \omega^{\prime} A_{\uparrow}^{2}\left(\epsilon, \omega^{\prime}\right) f\left(\omega^{\prime}\right)\left[1-f\left(\omega^{\prime}\right)\right],
$$

where $T$ is the temperature.

At $t=0$ and $U=2$ the dc-resistivity shows a semiconducting-like behavior with temperature. Above $T=0.6$, not shown, it starts to increase with $T$ as in a metal. In accordance with our previous results for the insulator-metal transition the static resistivity decreases as function of the transverse field. One can clearly see in Fig. 5 the presence of an isosbectic point, where all the curves crosses at the same temperature. It is important to mention that a similar behavior for the static resistivity as the one we obtain for $0.45<t<0.5$ has been observed in the normal state of $\mathrm{Bi}_{2} \mathrm{Sr}_{2} \mathrm{CuO}$.

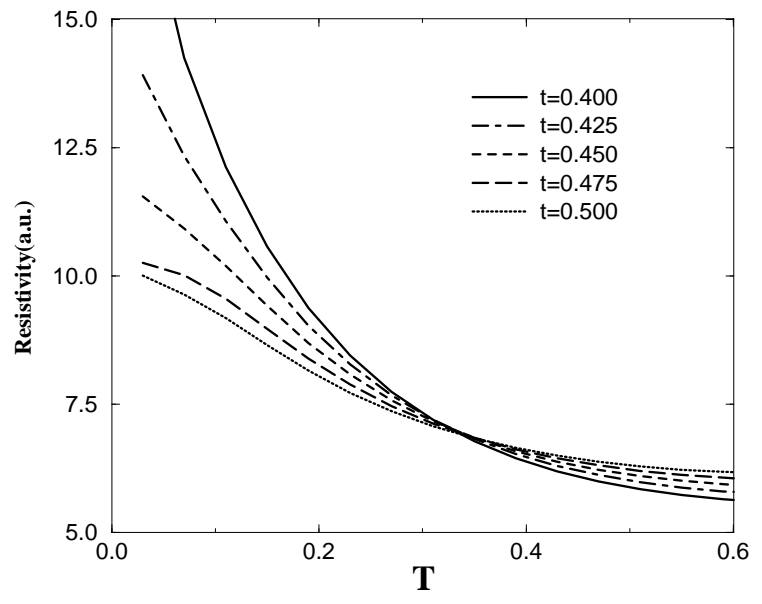

FIG. 5. Temperature dependence of the static resistivity in the half-filled SH model for $U=2$ and different values of $t$.

In Ref. [8] the in-plane $\left(\rho_{a b}\right)$ and out-of-plane $\left(\rho_{c}\right)$ dcresistivity of $\mathrm{Bi}_{2} \mathrm{Sr}_{2} \mathrm{CuO}$ have been measured for different values of the magnetic field. The experiment shows completely different behavior for the in-plane and out- of-plane measurements: $\rho_{a b}$ increases to a certain saturation value, while $\rho_{c}$ strongly decreases (by a factor $10^{4}$ ) for the same values of the magnetic field. One can understand this contrasting behavior taking into account our results and those of Ref. [24]. As mentioned before, it was shown in Ref. [24] that the critical $U$ for the metal-insulator crossover is reduced by the effect of a $z$-component magnetic field. Thus for the same value of $U$ the system loses its metallic phase as a function of the field and the resistivity increases with the Zeeman field. On the other hand, we show here that a field applied along to the $x$-direction (in the spin space) drives and insulator to a metal transition in the system. Since both theoretical results agree with was observed in the measurements of Ando et al., one can conclude that the direction along which the field is applied is essential in systems with broken spin symmetry.

\section{CONCLUSIONS}

We report the results of the first systematic study of a strongly correlated electron system in transverse fields, undertaken to understand the interplay between on-site correlations and local spin fluctuations. To make contact with experiment we consider the simplified Hubbard ( $\mathrm{SH}$ ) model, a model Hamiltonian with broken spin symmetry. The relevant one-particle Green's functions for this model in the presence of a transverse field $t$ are obtained by means of a perturbative treatment of the hopping and the field around the atomic limit. We employ the static approximation to study the insulator-metal transition as a function of $t$. At intermediate values of the field the metallic phase shows very interesting features. In this regime we observe the presence of sharp peaks in the spectral function of the heavy-electrons at the Fermi level. We show that the static approximation is very accurate in describing the dynamics of the heavy-particles in intermediate and strong values of $U$.

We have also studied the optical properties of the $\mathrm{SH}$ model as a function of the transverse field. Our results for the optical conductivity and static resistivity confirm the insulator-metal crossover. We found good qualitative agreement between our results for the latter quantity and those observed in the out-of-plane resistivity of $\mathrm{Bi}_{2} \mathrm{Sr}_{2} \mathrm{CuO}_{y}$ 目. In both theoretical and experimental investigations the resistivity decreases as a function of the magnetic field. In addition, we observe in our results the presence of an isosbectic point, where all curves cross at the same temperature. This crossing point seems to appear also in the experiment, although it could not be clearly observed because the measurements were done for temperatures slightly below the occurrence of the crossing.

To further our understanding about the semiconducting behavior along to the $c$-axis in cuprates 36 , we should mention that in the non-magnetic phase the $\mathrm{SH}$ model 
has an intrinsic disorder built into it. Such disorder is responsible for the non-Fermi liquid properties of the conduction electrons, since the heavy-electrons act as randomly distributed scattering centers. For large values of disorder ( $U$ in the present model) the motion of the conduction electrons is totally suppressed and the system behaves as a semiconductor, as shown in Fig. 5 for small values of $t$. According to our results, the semincoducting behavior can be partially suppressed by enhancing the local spin degrees of freedom through a transverse field. Following this scenario and the measurements of the $d c$ resistivity in $\mathrm{Bi}_{2} \mathrm{Sr}_{2} \mathrm{CuO}$ y one can suggest that the low temperature semiconducting behavior in cuprates along the $c$-direction might result from conventional random disorder.

Finally, as a possible extension of this work we should consider in the future the possibility of extending our approach to the case of the one-band Hubbard model. In this case it would be interesting to analyze the effect of the transverse field on the well known Fermi-liquid properties of this model. Furthermore, inspired by the results of Fig. 1 it would be interesting to analyze whether our perturbation method for the local problem is also accurate in describing the single particle properties of the Hubbard model in the large $U$ limit. Unfortunately, apparently there are no exact results for this model at $T=0$ in $d=\infty$, but one can follow the spirit of Ref. [29] and compare our results for the Matsubara Green's functions with those of finite temperature quantum Monte Carlo simulations 26 . We believe that the static approximation (Eq. (5)) is an effective approximation for the Hubbard model if we consider that the local spin fluctuation are irrelevant in the strong coupling limit.

\section{ACKNOWLEDGMENTS}

The author wishes to acknowledge M. Foglio, C. Kuebert and P. Farinas for useful discussions and comments. This work was supported by the Fundação de Amparo à Pesquisa do Estado de São Paulo (FAPESP).

${ }^{1}$ D. Goldhaber-Gordon et al. Nature 391, 156 (1998); H. Imamura et al. Physica B 256-258, 194 (1998); M. Pustilnik et al. preprint con-mat/9908004.

2 S. M. Cronenwett et al., Science 281, 540 (1998).

${ }^{3}$ H. D. Drew et al. J. Phys. Cond. Mat. 8, 10037 (1996).

${ }^{4}$ H. T. S. Lihn et al. Phys. Rev. Lett. 76, 3810 (1996).

${ }^{5}$ L. S. Uspenskaya et al. Phys. Rev. B 56, 11979 (1997).

${ }^{6}$ J. Buan et al. Phys. Rev. Lett. 72, 2632 (1994); E. H. Brandt Phys. Rev. Lett. 74, 3025 (1995); A. V. Kuznetsov et al. Phys. Rev B 529637 (1995); M. Wurlitzer et al. Phys. Rev. B 55, 11816 (1997).
${ }^{7}$ G. S. Boebinger et al. Phys. Rev. Lett. 77, 5417 (1996).

${ }^{8}$ Y. Ando et al. Phys. Rev. Lett. 77, 2065 (1996).

${ }^{9} \mathrm{P}$. W. Anderson in "The Theory of Superconductivity in the High- $T_{c}$ cuprates", Princeton University Press (1997).

${ }^{10}$ M. Imada et al. Rev. Mod. Phys. 70, 1039 (1999).

${ }^{11}$ D. Vaknin et al. Phys. Rev. Lett. 58, 2802 (1987); E. Brecht et al. Phys. Rev. B 52, 9601 (1995).

${ }^{12}$ K. Terakura et al. Phys. Rev. B 30, 4734 (1984); W. Bao et al. Phys. Rev. Lett 71, 766 (1993); G. Blumberg et al. Phys. Rev. Lett. 80, 564 (1998).

${ }^{13}$ B. Batlogg, in "The Los Alamos Symposium" eds. R. Coffey et al. Addison Wesley Publ. (1991).

${ }^{14}$ C. L. Seaman et al. Phys. Rev. Lett. 67, 2882 (1991); H. V. Löhneysen et al. Phys. Rev. Lett. 72, 3262 (1994); L. Degiorgi et al. Phys. Rev. B 52, 42 (1995); M. B. Maple et al. Physica B 223-224, 447 (1996); F. Bommeli et. al. Phys. Rev. B 5610001 (1997).

15 J. Voit Rep. Prog. Phys. 58, 977 (1995).

${ }^{16}$ Q. Si et al., Phys. Rev. Lett. 72, 2761 (1994).

${ }^{17}$ R. Consiglio and M. A. Gusmão, Phys. Rev. B 55, 6825 (1997).

18 U. Brandt and C. Mielsch, Z. Phys. B 75, 365 (1989).

${ }^{19}$ V. Janiš, Z. Phys. B 83, 227 (1991).

${ }^{20}$ Q. Si it et al., Phys. Rev. B 46, 1261 (1992).

${ }^{21}$ P. de Vries et al. Z. Phys. B 92, 353 (1993).

${ }^{22}$ P. Farkašovský, Phys. Rev. B 51, 1507 (1995).

${ }^{23}$ U. Brandt and M. P. Urbanek, Z. Phys. B 89, 297 (1992).

${ }^{24}$ P. G. J. van Dongen and C. Leinung, Annalen der Physik 6, 45 (1997).

${ }^{25}$ W. Metzner and D. Vollhardt, Phys. Rev. Lett. 62, 324 (1989); see also E. Müller-Hartmann, Z. Phys. B 74, 507 (1989).

26 A. Georges et al., Rev. Mod. Phys. 68, 13 (1996).

${ }^{27}$ L. Craco and M. A. Gusmão, Phys. Rev. B 54, 1629 (1996).

${ }^{28}$ N. E. Bonesteel et al., Phys. Rev. Lett. 68, 2684 (1992); T. Yildirim et al., Phys. Rev. Lett. 73, 2919 (1994).

${ }^{29}$ L. Craco, Phys. Rev. B 59, 14837 (1999).

${ }^{30}$ L. Craco and M. A. Gusmão, Phys. Rev. B 52, 17135 (1995).

31 The spin degeneracy can be lifted by a strong magnetic field or by very large Hund's-rule coupling, as in the case of $\mathrm{Fe}_{3} \mathrm{O}_{4} \mathrm{B2}$.

32 J. R. Cullen and E. Callen, Phys. Rev. Lett. 26, 236 (1971).

${ }^{33}$ More precisely, $\bar{g}_{0 \sigma}\left(i \omega_{n}\right) \equiv 1 /\left(i \omega_{n}-E+\mu-\mathcal{A}_{\sigma}\left(i \omega_{n}\right)\right)$ and $\bar{g}_{\bar{\sigma} 2}\left(i \omega_{n}\right) \equiv 1 /\left(i \omega_{n}-E-U+\mu-\mathcal{A}_{\sigma}\left(i \omega_{n}\right)\right)$.

34 The calculations were performed at the symmetric case with a Gaussian DOS.

${ }^{35}$ G. Möller et al. Phys. Rev. B 46, 7427 (1992).

${ }^{36}$ Y. Iye, in "Physical Properties of High Temperature Superconductors $I I I$, edited by D. M. Ginsberg (World Scientific, Singapore, 1991). 